\title{
Biologia reprodutiva de três espécies simpátricas de peixes neotropicais: Pimelodus maculatus Lacépède (Siluriformes, Pimelodidae), Leporinus amblyrhynchus Garavello \& Britski e Schizodon nasutus Kner (Characiformes, Anostomidae) do recém-formado Reservatório de Miranda, Alto Paraná
}

\author{
Volney Vono ${ }^{1}$ \\ Luiz Gustavo M. Silva ${ }^{2}$ \\ Bruno P. Maia ${ }^{2}$ \\ Hugo P. Godinho ${ }^{3}$
}

\begin{abstract}
Reproductive biology of three simpatric species of Neotropical fishes: Pimelodus maculatus Lacépède (Siluriformes, Pimelodidae), Leporinus amblyrhynchus and Schizodon nasutus Kner (Characiformes, Anostomidae) from the newly formed Miranda Reservoir, High Paraná Basin. The reproductive activity parameters of the teleosts Pimelodus maculatus Lacépède, 1803, Leporinus amblyrhynchus Garavello \& Britski, 1987 and Schizodon nasutus Kner, 1859 was evaluated at a newly formed reservoir in the Araguari River, Paraná River basin, Southeast Brazil. The fishes were captured by gill nets bimonthly from November 1997 to November 1998 at three sampling stations. The stages of gonad development were identified macroscopically in the field and then confirmed by histological analysis in the laboratory. The females of all species reached larger standard length than the males. Pimelodus maculatus in reproductive activity were captured only in November, 1997 and January, 1998 whereas L. amblyrhynchus and S. nasutus were in activity throughout the year. The gonadosomatic index reached the peak during the advanced maturation stage in all species, but the hepatosomatic index and coelomic fat index exhibited distinct tendencies. Length at first maturity was $9.5 \mathrm{~cm}$ SL for $L$. amblyrhynchus females and 17.1 and $13.0 \mathrm{~cm} \mathrm{SL}$, respectively, for $S$. nasutus females and males. L. amblyrhynchus and $S$. nasutus are partial spawners. The size at first maturity and type of spawning of $P$. maculatus were not possible to determine in this work.
\end{abstract}

KEY WORDS. Fish reproduction, reservoir, dam effects

Ao longo das últimas décadas, a rica diversidade da ictiofauna do sudeste do Brasil tem sido fortemente submetida à ações antrópicas, especialmente em função da construção de grande número de barragens hidrelétricas. Essas barragens e seus respectivos reservatórios alteram o curso natural dos rios, modificando sua feição lótica com consequentes perdas e surgimentos de novos habitats.

1) Programa de Pós-Graduação em Ecologia, Conservação e Manejo de Vida Silvestre, Universidade Federal de Minas Gerais. Avenida Antonio Carlos 6627, Caixa Postal 486, 31270-901 Belo Horizonte, Minas Gerais, Brasil.

2) Bolsista FIP/PUC Minas.

3) Programa de Pós-graduação em Zoologia de Vertebrados, PUC Minas Gerais. Avenida Dom José Gaspar 500, Prédio 41, 30535-610 Belo Horizonte, Minas Gerais, Brasil.

Revta bras. Zool. 19 (3): 819 - 826, 2002 
A habilidade reprodutiva dos peixes sujeitos à essa nova situação determinará, dentre outras condições, o sucesso de sua colonização no novo ambiente. Espera-se que espécies com alta plasticidade quanto aos habitats de desova estejam entre os colonizadores de maior sucesso (revisão em AGOSTINHO et al. 1999). Dessa forma, estudos de biologia reprodutiva de peixes em reservatórios recém-formados podem fornecer dados sobre sua nova condição no ambiente, subsidiando estratégias de conservação e manejo.

As espécies analisadas foram o mandi-amarelo Pimelodus maculatus Lacépède, 1803, o timburé Leporinus amblyrhynchus Garavello \& Britski, 1987 e a taguara Schizodon nasutus, Kner, 1859, as quais estão entre as mais abundantes, numericamente, no reservatório de Miranda. O mandi-amarelo tem ampla distribuição nas bacias hidrográficas brasileiras (GODOY 1975; BURGESS 1989) e, em algumas dessas, é importante nas pescarias profissional e esportiva (FERREIRA et al. 1997; PETRERE 1989). As informações de literatura sobre sua atividade migratória e tipo de desova são controversas. GARAVELLO \& BRITSKI (1987) realataram a ocorrência do timburé para a bacia do rio Paraná. No reservatório de Furnas (Rio Grande), seu período reprodutivo é prolongado e a desova é parcelada (RICARDO et al. 1997). A taguara é endêmica da bacia do Paraná (GARAVELLO \& BRITSKI 1990), tendo sido considerada migradora por GODOY (1975). O presente trabalho visa avaliar a atividade reprodutiva destas três espécies no recém-formado reservatório de Miranda, no rio Araguari, com respeito à época e o tipo de desova, às relações ponderais entre vísceras e vitelogênese e ao tamanho de primeira maturação para ambos os sexos.

\section{MATERIAL E MÉTODOS}

O reservatório de Miranda ( $19^{\circ} 00^{\prime} \mathrm{S}$ e $\left.48^{\circ} 00^{\prime} \mathrm{W}\right)$ localiza-se no rio Araguari, afluente do rio Paranaíba, bacia do Paraná Superior, sendo limitado a montante pela barragem de Nova Ponte e a jusante por trecho livre de rio de cerca de $100 \mathrm{~km}$ de extensão até o reservatório de Itumbiara (Fig. 1). Seu enchimento se deu em agosto de 1997. Possui área inundada de $50,6 \mathrm{~km}^{2}$, profundidade máxima de $75 \mathrm{~m}$ e armazena até 1,145 bilhões de $\mathrm{m}^{3}$ de água. O nível máximo de depleção alcança $3 \mathrm{~m}$.

As coletas foram realizadas nos meses de novembro de 1997 e de janeiro, março, maio, agosto e novembro de 1998 em três pontos do reservatório de Miranda (Fig. 1). Para a captura dos peixes utilizaram-se redes de emalhar de malhas variando de 3,0 a $16,0 \mathrm{~cm}$, entre nós opostos, armadas ao entardecer e retiradas na manhã seguinte, permanecendo expostas por cerca de 14 horas. Os peixes foram identificados, etiquetados e fixados em solução de formol a $10 \%$ e posteriormente transferidos para solução de álcool a $70^{\circ} \mathrm{GL}$.

Obtiveram-se os dados de comprimento padrão, pesos corporal, das gônadas, do fígado e da gordura celômica e a determinação do sexo. Através da análise macroscópica dos ovários obtidas em campo, determinaram-se os estádios de maturação gonadal de acordo com as seguintes características: 1) repouso: ovários delgados e íntegros, sem ovócitos visíveis a olho nú; 2) em maturação: ovários com discreto aumento de volume e ovócitos evidentes; 3 ) maduro: ovários com volume 




Fig. 1. Localização da área de estudos e pontos de amostragem.

máximo e ovócitos vitelogênicos distribuídos uniformemente; e 4) total ou parcialmente desovado: ovários flácidos e sanguinolentos, com número variável de ovócitos vitelogênicos remanescentes. Em laboratório, gônadas em estádios de maturação duvidosos foram reavaliadas através de análise microscópica.

Determinou-se o ciclo reprodutivo de cada espécie a partir da distribuição da freqüência relativa de seus estádios de maturação gonadal obtida nos períodos amostrais. Foram calculados os índices gonadossomático (IGS = PG/PC*100), hepatossomático (IHS = PF/PC*100) e de gordura celômica (IGC = PGC/PC*100), sendo $\mathrm{PG}=$ peso da gônada, $\mathrm{PF}=$ peso do fígado, $\mathrm{PGC}=$ peso da gordura celômica e $\mathrm{PC}=$ peso corporal.

O tamanho de primeira maturação foi estimado considerando-se aquele do menor indivíduo capturado em atividade reprodutiva. O número de classes de tamanho dos peixes foi obtido segundo SAMPAIO (1998). Aplicou-se o teste do $X^{2}$ (nível de 5\%) para verificação da hipótese da segregação 1:1 entre machos e fêmeas das três espécies em estudo.

\section{RESULTADOS}

As freqüências absoluta e relativa por classe de comprimento padrão para machos e fêmeas de mandi-amarelo, timburé e taguara encontram-se nas tabelas I, II e III, respectivamente. Para as três espécies, as fêmeas alcançaram comprimentos maiores que os machos. Para o mandi-amarelo e a taguara verificou-se que a segregação de sexos na população obedeceu à relação $1: 1(\mathrm{p}<5 \%)$, enquanto que para o timburé tal relação não foi registrada. 
Tabela I. Freqüência absoluta $(\mathrm{Fa})$ e relativa ( $\mathrm{Fr})$, por classe de comprimento padrão de machos e fêmeas de mandi-amarelo Pimelodus maculatus no reservatório de Miranda, em 1997-1998.

\begin{tabular}{|c|c|c|c|c|}
\hline \multirow{2}{*}{ Classe $(\mathrm{cm})$} & \multicolumn{2}{|c|}{ Fêmeas } & \multicolumn{2}{|c|}{ Machos } \\
\hline & Frequêencia absoluta & Freqüência relativa & Freqüência absoluta & Freqūência relativa \\
\hline $14,2-16,9$ & 2 & 11,1 & 16 & 88,9 \\
\hline $17,0-19,7$ & 13 & 28,3 & 33 & 71,7 \\
\hline $19,8-22,5$ & 14 & 42,4 & 19 & 57,6 \\
\hline $22,6-25,3$ & 19 & 90,5 & 2 & 9,5 \\
\hline $25,4-28,1$ & 13 & 100,0 & 0 & 0,0 \\
\hline $28,2-30,9$ & 10 & 100,0 & 0 & 0.0 \\
\hline $31,0-33,7$ & 3 & 100,0 & 0 & 0,0 \\
\hline $33,8-36,5$ & 1 & 100,0 & 0 & 0,0 \\
\hline $36,6-39,3$ & 1 & 100,0 & 0 & 0,0 \\
\hline Total & 76 & & 70 & \\
\hline
\end{tabular}

Tabela II. Freqüência absoluta $(\mathrm{Fa})$ e relativa $(\mathrm{Fr})$, por classe de comprimento padrão para machos e fêmeas de timburé Leporinus amblyrhynchus no reservatório de Miranda, em 1997-1998.

\begin{tabular}{|c|c|c|c|c|}
\hline \multirow{2}{*}{ Classe $(\mathrm{cm})$} & \multicolumn{2}{|c|}{ Fêmeas } & \multicolumn{2}{|c|}{ Machos } \\
\hline & Freqüência absoluta & Freqüência relativa & Freqüência absoluta & Freqüência relativa \\
\hline $9,5-10,3$ & 7 & 63,6 & 4 & 36,4 \\
\hline $10,4-11,2$ & 15 & 75,0 & 5 & 25,0 \\
\hline $11,3-12,1$ & 24 & 63,2 & 14 & 36,8 \\
\hline $12,2-13,0$ & 24 & 72,7 & 9 & 27,3 \\
\hline $13,1-13,9$ & 13 & 65,0 & 7 & 35,0 \\
\hline $14,0-14,8$ & 4 & 100,0 & 0 & 0,0 \\
\hline $14,9-15,7$ & 4 & 100,0 & 0 & 0,0 \\
\hline $15,8-16,6$ & 1 & 100,0 & 0 & 0,0 \\
\hline Total & 92 & 70,2 & 39 & 29,8 \\
\hline
\end{tabular}

Tabela III. Freqüência absoluta $(\mathrm{Fa})$ e relativa $(\mathrm{Fr})$, por classe de comprimento padrão para machos e fêmeas de taguara Schizodon nasutus no reservatório de Miranda, em 1997-1998.

\begin{tabular}{|c|c|c|c|c|}
\hline \multirow{2}{*}{ Classe $(\mathrm{cm})$} & \multicolumn{2}{|c|}{ Fêmeas } & \multicolumn{2}{|c|}{ Machos } \\
\hline & Freqüência absoluta & Freqüência relativa & Freqüência absoluta & Freqüência relativa \\
\hline $13,0-15,1$ & 0 & 0,0 & 11 & 100,0 \\
\hline $15,2-17,3$ & 1 & 10,0 & 9 & 90,0 \\
\hline $17,4-19,5$ & 4 & 40,0 & 6 & 60,0 \\
\hline $19,6-21,7$ & 10 & 37,0 & 17 & 63,0 \\
\hline $21,8-23,9$ & 20 & 64,5 & 11 & 35,5 \\
\hline $24,0-26,1$ & 17 & 80,9 & 4 & 19,1 \\
\hline $26,2-28,3$ & 23 & 92,0 & 2 & 8,0 \\
\hline $28,4-30,5$ & 7 & 100,0 & 0 & 0.0 \\
\hline $30,6-32,7$ & 1 & 100,0 & 0 & 0,0 \\
\hline Total & 83 & 58,0 & 60 & 42,0 \\
\hline
\end{tabular}


O período de atividade reprodutiva das fêmeas variou entre as espécies. Fêmeas de mandi-amarelo em atividade reprodutiva foram capturadas apenas nas coletas de novembro de 1997 e janeiro de 1998, época coincidente com o período chuvoso. Para o timburé, a atividade reprodutiva estendeu-se por quase todos os meses de coleta, com exceção do mês de maio de 1998. Quanto à taguara, capturaram-se fêmeas em atividade reprodutiva nos meses de novembro de 1997 e janeiro, agosto e novembro de 1998 (Fig. 2).

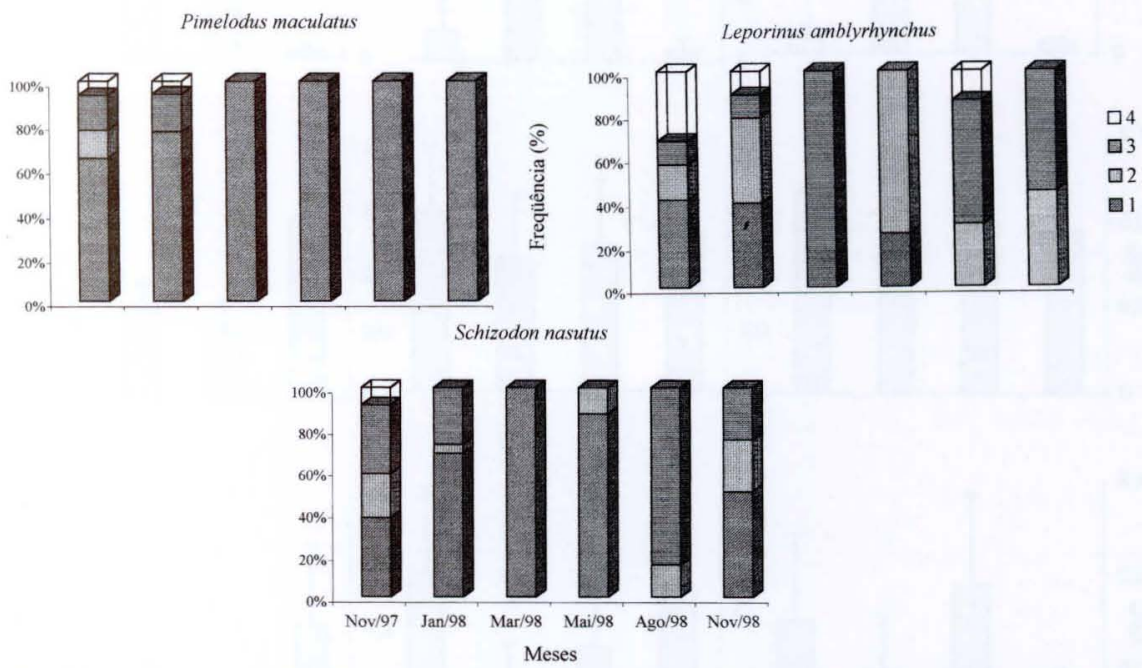

Fig. 2. Freqüência relativa dos estádios de maturação gonadal $(Y, \%)$ de fêmeas de mandiamarelo Pimelodus maculatus, do timburé Leporinus amblyrhynchus e da taguara Schizodon nasutus do reservatório de Miranda, rio Araguari, em 1997-1998. Estádios: (1) repouso, (2) em maturação, (3) maduro, (4) total ou parcialmente desovado.

O IGS variou ao longo dos estádios do ciclo reprodutivo, com valores máximos variando de 9,2, 21,3 e 13,5 para mandi-amarelo, timburé e taguara, respectivamente.. Embora o IHS tenha se alterado pouco ao longo do ciclo reprodutivo, no mandi-amarelo ele acompanhou a tendência do IGS com maior valor no estádio 3. Por outro lado, no timburé, o IHS mostrou tendência contrária ao do IGS, tendo o menor valor no estádio 3. Na taguara, o IHS não apresentou tendência perceptível ao longo do ciclo reprodutivo. O IGC apresentou queda nos estádios de maturação gonadal 2 e 3 no timburé e na taguara. No mandi-amarelo, as variações do IGC não indicaram nenhuma tendência clara em relação aos estádios de maturação gonadal (Fig. 3).

Para machos e fêmeas de mandi-amarelo não foi possível estabelecer o tamanho de primeira maturação, pois os menores indivíduos capturados já se encontravam em atividade reprodutiva. Para fêmeas de timburé, o tamanho de primeira maturação foi de $9,5 \mathrm{~cm}$ CP; para machos não foi possível estabelecer este parâmetro pela mesma razão indicada acima. Para a taguara, o tamanho de primeira maturação foi de 17,1 cm CP para fêmeas e 13,0 cm CP para machos. 


\section{Pimelodus maculatus Leporinus amblyrhynchus Schizodon nasutus}
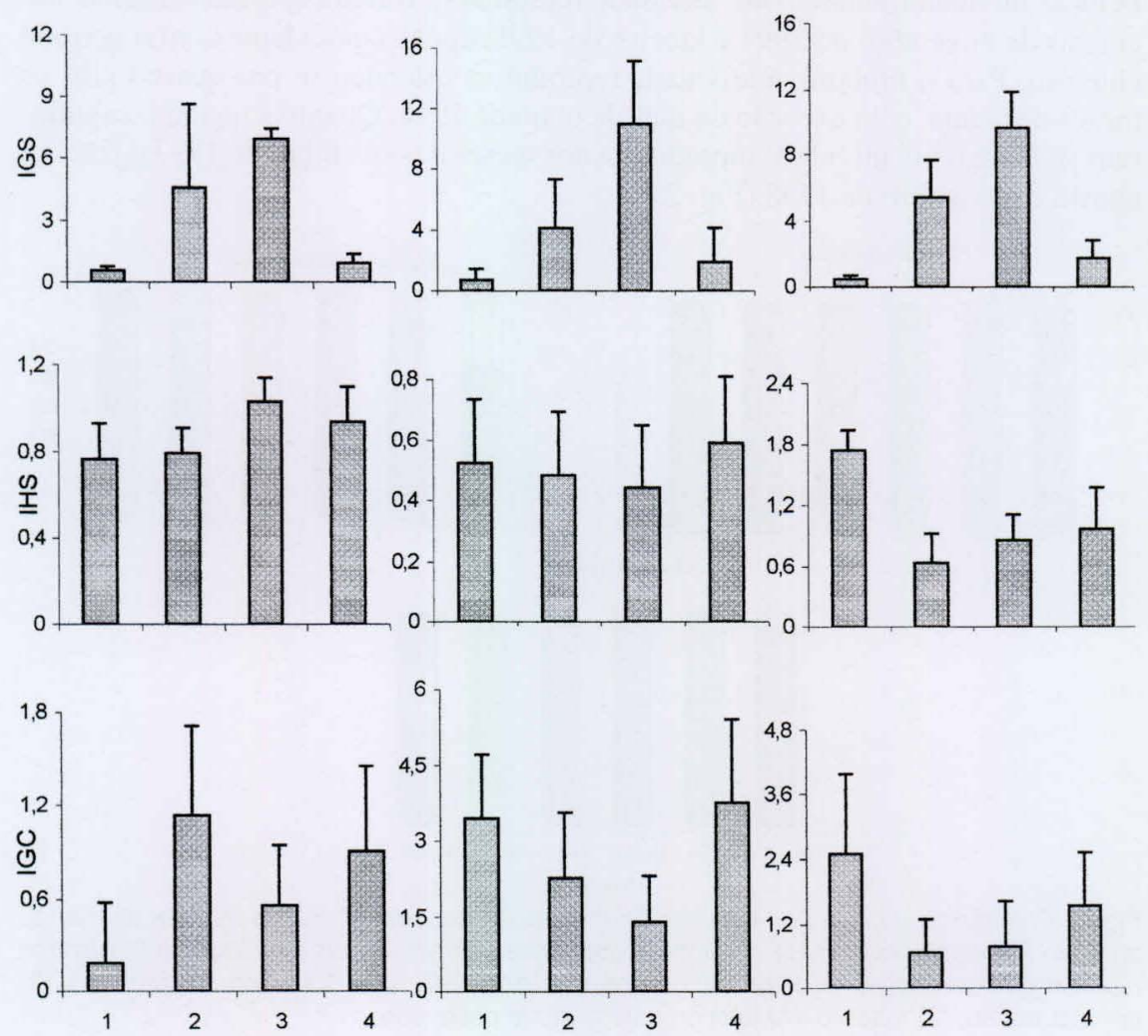

Estádio de maturação gonadal

Fig. 3. Índices gonadossomático (IGS), hepatossomático (IHS) e de gordura celômica (IGC) por estádio de maturação gonadal de fêmeas do mandi-amarelo Pimelodus maculatus, do timburé Leporinus amblyrhynchuse da taguara Schizodon nasutus no reservatório de Miranda, rio Araguari, em 1997-1998. Barras verticais indicam desvio padrão.

\section{DISCUSSÃO}

Fêmeas de mandi-amarelo em atividade reprodutiva foram capturadas apenas nos meses de novembro de 1997 e janeiro de 1998. Este dado pode indicar que esses exemplares encontravam-se em maturação gonadal no momento do fechamento da barragem, ocorrido em agosto de 1997. Por outro lado, a ausência de fêmeas em maturação gonadal e desovadas no ano seguinte (1999) sugere que o novo ambiente não ofereceu condições para o desenvolvimento do ciclo reprodutivo dessa espécie.

Assim como registrado por RICARDO et al. (1997) no reservatório de Furnas (rio Grande, Paraná Superior), o timburé apresentou atividade reprodutiva ao longo de todo $o$ ano no reservatório de Miranda. A exemplo do timburé, a taguara também 
apresentou atividade reprodutiva durante praticamente todo o ano, com maior intensidade no mês de agosto. Por outro lado, GoDOY (1975) registrou atividade reprodutiva da taguara entre os meses de novembro e janeiro, considerando-a como migradora no rio Mogi-Guassu, São Paulo.

Variações ambientais ou diferentes metodologias utilizadas para se determinar o período reprodutivo podem levar a resultados conflitantes (BAzzOLI et al. 1997), tal como parece acontecer com as espécies em estudo. Cabe salientar que, na ocasião das coletas, o reservatório de Miranda consistia em ambiente recém-formado, no qual suas populações de peixes possivelmente ainda se encontravam sob as influências das condições lóticas do segmento do rio Araguari.

Para a taguara e o timburé, não há dados disponíveis na literatura sobre o tamanho de primeira maturação, portanto, os valores aqui estimados são os primeiros a serem obtidos.

A segregação 1:1 entre machos e fêmeas é, em geral, encontrada em populações de peixes, assim como registrado neste trabalho para o mandi-amarelo e a taguara. Entretanto, ocorrem variações na proporção sexual em função de mortalidade, crescimento e comportamento das espécies (VAZZOLER 1996). As freqüências por classe de comprimento das referidas espécies indicaram que fêmeas predominaram nas classes superiores.

Com exceção do mandi-amarelo, as três espécies aparentemente desovaram no reservatório de Miranda durante o período de estudo. Em função do longo período reprodutivo e do número expressivo de ovócitos vitelogênicos remanescentes no estádio desovado do timburé e da taguara, sugere-se que o tipo de desova dessas espécies seja parcelado tal como já registrado para o timburé (RICARDO et al. 1997).

Dados obtidos em ambientes naturais (GoDINHO et al. 1974; BAZZOLI et al. 1997; VAZZOLER 1996; AGOSTINHO \& JÚLIO 1999) indicaram que o mandi-amareloa espécie apresenta desova parcelada. No entanto, o número reduzido de fêmeas desovadas coletadas não permite inferências a esse respeito.

Quanto à capacidade migradora, o mandi-amarelo foi considerado migrador de longa distância (VAZzOLER 1996; VAzZOLER et al. 1997; AgOsTinho et al. 1999), embora possa realizar deslocamentos menores do que aqueles efetuados por migradores de longa distância (AGOSTINHO \& Júlio 1999). Indivíduos de mandiamarelo em atividade reprodutiva, incluindo fêmeas desovadas, foram registrados em reservatórios do rio Grande (BAZzOLI et al. 1997).

Dados conclusivos a respeito da atividade reprodutiva dessa espécie, no reservatório de Miranda, poderiam ser obtidos somente após um maior período amostral. No entanto, adianta-se que a possibilidade da espécie migrar para ambientes adjacentes é nula, pois os poucos tributários existentes são de dimensões muito reduzidas e, na maioria dos casos, apresentando desníveis acentuados, muito acima do nível do reservatório.

As análises de IHS e IGC não apontam uma relação direta com respeito à participação do fígado e da gordura celômica no processo de vitelogênese do mandi-amarelo. Quanto ao timburé, os dados são sugestivos dessa participação pois o IHS reduziu-se gradualmente ao longo do processo de maturação do ovário. Da 
mesma forma, os dados obtidos para IGC do timburé indicam redução das reservas graxas durante o período reprodutivo. Para a taguara, apenas as reservas graxas aparentemente reduziram-se durante o período reprodutivo.

AGRADECIMENTOS. Agradecemos ao GE/PA, da Companhia Energética de Minas Gerais (CEMIG), pelo suporte financeiro dado ao estudo, ao CNPq e ao FIP PUC MINAS pelas bolsas concedidas. À Denise da Silveira Lemos, pelo auxílio na dissecção dos indivíduos de taguara.

\section{REFERÊNCIAS BIBLIOGRÁFICAS}

Agostinho, A.A.; L.E. Miranda; L.M. Bini; L.C. Gomes; S.M. Thomaz \& H.I. Suzuky. 1999. Patterns of colonization in neotropical reservoirs, and prognoses on aging, p. 227-265. In: J.G. TUNDISI \& M. STRASKRaba (Eds). Theorical reservoir ecology and its applications. Leiden, Backhuys Publishers, 592p.

Agostinho, A.A. \& H.F. Júlıo JR. 1999. Peixes da bacia do alto rio Paraná, p. 374-400. In: R.H. Lowe-McConnell (Ed.). Estudos ecológicos de comunidades de peixes tropicais. São Paulo, Brasil, Edusp, 535p.

Bazzoli, N.; L.C.V. Cangussu; E. Rizzo \& G.B. Santos. 1997. Reprodução e desova de mandis Pimelodus maculatus e Iheringichthys labrosus (Pisces, Pimelodidae) nos reservatórios de Furnas, Marimbondo e Itumbiara. Bios, Belo Horizonte, 5 (5): 7-15.

BURGESS, W.E. 1989. An atlas of freshwater and marine catfishes. A preliminary survey of the Siluriformes. Neptune City, FH Publications Inc., 784p.

Ferreira, A.O; V. Vono; F. Vieira \& C.B.M. Alves. 1997. Monitoramento do desembarque pesqueiro no reservatório da UHE Nova Ponte, p. 1-7. In: Anais do XIV SNPTEE - Seminário Nacional de produção e transmissão de energia elétrica, Belém, 112p.

Garavello, J.C. \& H.A. Britski. 1987. Duas novas espécies do gênero Leporinus Spix, 1829 da bacia do Alto Paraná, (Teleostei, Anostomidae). Com. Mus. Ciênc. PUCRS, Porto Alegre, 44: 153-165. . 1990. Duas novas espécies do gênero Schizodon Agassiz da bacia do Alto Paraná, Brasil, América do Sul (Ostariophisy, Anostomidae). Naturalia, São Paulo, 15: 153-170.

GodinHo, H.M.; S. FERRI; L.O. MEDEIROS \& J.M.B. BARKER. 1974. Morphological changes in the ovary of Pimelodus maculatus Lacépède, 1803 (Pisces, Siluroidei), related to the reproductive cycle. Rev. Brasil. Biol., Rio de Janeiro, 34 (4): 581-588.

Godoy, M.P. 1975. Peixes do Brasil, subordem Characoidei-Bacia do Rio Mogi Guassu. Piracicaba, Franciscana, Vol. 4, 846p.

Petrere JR., M. 1989. River fisheries in Brazil: a review. Regulated Rivers Research and Management, Chichester, 4 (1): 1-16

Ricardo, M.C.P.; G.B. Santos; E. Rizzo \& N. Bazzoli. 1997. Aspectos reprodutivos de Leporinus amblyrhynchus Garavello \& Britski, 1987 e Leporinus striatus Kner, 1859 (Pisces: Anostomidae) no reservatório de Furnas, MG. Bios, Belo Horizonte, 5 (5): 29-35.

SAMPAIO, I.B.M. 1998. Estatística aplicada à experimentação animal. Belo Horizonte, Fundação de Ensino e Pesquisa em Medicina Veterinária e Zootecnia, 221p

VAZzoler, A.E.A.M. 1996. Biologia da Reprodução de Peixes teleósteos: teoria e prática. Maringá, EDUEM, 169p.

Vazzoler, A.E.A.M.; H.I. Suzuki; E.E. MARQues \& M.P. Lizama. 1997. Primeira maturação gonadal, períodos e áreas de reprodução, p. 249-265. In: A.E.A.M. VAzzoler; A.A. AgostinHo \& N.S. HAHN (Eds). A planície de inundação do Alto rio Paraná: aspectos físicos, biológicos e socioeconômicos. Maringá, EDUEM, 460p.

Recebido em 24.VIII.2001; aceito em 02.IX.2002.

Revta bras. Zool. 19 (3): 819 - 826, 2002 